DOI:10.15171/ipp.2019.03

\title{
Association of CDX2 expression and overall survival and clinicopathologic disease manifestations in patients with gastric cancer in Northwest of Iran
}

\author{
Farideh Akbari ${ }^{\circledR}$, Zohreh Ghoreishi ${ }^{2}$, Mohammad Asghari Jafarabadi ${ }^{3,4}$, Khatereh Rezazadeh ${ }^{2}$, Babak Nejati ${ }^{1}$, \\ Ali Esfahani ${ }^{1 *(\mathbb{D})}$,
}

${ }^{1}$ Hematology and Oncology Research Center, Tabriz University of Medical Sciences, Tabriz, Iran

${ }^{2}$ Nutrition Research Center, Department of Clinical Nutrition, School of Nutrition \& Food Sciences, Tabriz University of

Medical Sciences, Tabriz, Iran

${ }^{3}$ Road Traffic Injury Research Center, Tabriz University of Medical Sciences, Tabriz, Iran

${ }^{4}$ Department of Statistics and Epidemiology, Faculty of Health, Tabriz University of Medical Sciences, Tabriz, Iran

\section{*Correspondence to \\ Ali Esfahani, \\ Email: \\ ali.sfhn@gmail.com, \\ esfahania@tbzmed.ac.ir}

Received 3 January 2019 Accepted 6 February 2019 Published online 23 February 2019

Keywords: Gastric cancer, Homeobox protein CDX2, Overal survival, Clinicopathologic characteristics

\begin{abstract}
Introduction: In the northwest of Iran, gastric cancer is the first and third common form of malignancy in men and women, respectively. Homeobox protein CDX2, was proposed as a prognostic biomarker in gastric cancer.

Objectives: This study aimed to assess the association of CDX2 expression overall survival as well as clinicopathologic variables in patients with gastric cancer.

Materials and Methods: CDX2 expression was examined by the immunohistochemistry method in the formalin-fixed paraffin-embedded surgical specimens of gastric cancer. The association between CDX2 expression and overall survival, stage of the disease, metastasis status, pathology type of the tumor, and serum levels of tumor markers serum levels of tumor markers carcinoembryonic antigen (CEA), cancer antigen 125 (CA-125), cancer antigen 19-9 (CA 19-9) was then evaluated.

Results: Ninety-two patients with primary gastric cancer were recruited. CDX2 expression was positive in $72(78 \%)$ of the subjects. Overall survival was not significantly different between two groups of patients (CDX2 positive versus negative). However, the risk of death was $21 \%$ lower in CDX2 positive patients, after adjusting for age and gender $(P=0.565)$. Disease stage and metastasis rate were significantly lower in CDX2 positive patients $(P=0.027$, and $P=0.021$, respectively). Additionally, $71 \%$ of the CDX2 positive subjects had intestinal pathology type of the disease $(P=0.025)$. Accordingly, a significant lower level of CA19-9 was found in CDX2 positive patients as well $(P=0.002)$.

Conclusion: There was not any significant association between CDX2 expression and overall survival in patients with gastric cancer, although the risk of death was lower in CDX2 positive subjects. However, there was a less invasive pattern of gastric carcinoma in this group of patients.
\end{abstract}

\section{Introduction}

Gastric cancer is the fourth most common malignancy throughout the world after lung, breast and colorectal neoplasms, and the second and fourth reason of malignancy associated death in men and women, respectively (1). According to world health organization GLOBOCAN 2012 database, gastric cancer is the most common malignancy in men and the third one in women after breast and colorectal cancers in Iran, and also in East Azerbaijan province in the northwest, where this study was conducted. Gastric cancer is the most common cancer in men and the third one in women after skin and colorectal cancers (2-4).

\section{Key point}

In a study on 92 patients with primary gastric cancer, homeobox protein CDX2 expression did not show any significant association with overall survival. However, CDX2 positive patients had lower risk of death compared with CDX2 negative patients.

CDX2 protein, an intestinal transcription factor, is encoded by the CDX2 gene, known as caudal type homeobox 2 (5). It is expressed in the intestinal epithelial cells from duodenum to the rectum and has an essential role in intestinal epithelial cells' production and differentiation (6). CDX2 is considered as an important prognostic

\footnotetext{
Copyright (C) 2019 The Author(s); Published by Nickan Research Institute. This is an open-access article distributed under the terms of the Creative Commons Attribution License (http://creativecommons.org/licenses/by/4.0), which permits unrestricted use, distribution, and reproduction in any medium, provided the original work is properly cited.
} 
biomarker in gastric cancer and several studies showed that there is a positive association between CDX2 and favorable treatment outcomes including longer survival (7-10). However, Studies on the association between CDX2 expression and treatment outcomes in patients with gastric cancer are controversial. In some studies, CDX2 positive gastric cancerous tissues were more resectable resulting in a higher rate of patients' survival (7) and it was identified as a marker for better prognosis $(11,12)$. On the other hand, there were studies showed that representation of CDX2 was related to the development of intestinal metaplasia proportionally (12-15).

Despite the ongoing decline in the incidence of gastric cancer throughout the world, it is expected to increase by more than $50 \%$ by 2030 in Iran based on World Health Organization GLOBOCAN database. Moreover, gastric cancer is still very fatal, and the patients have a low rate of survival and poor prognosis, mainly due to the late diagnosis in advanced stages of the disease. Moreover, there are no strong prognostic factors for predicting outcomes in those patients who have more critical conditions and needs more medical and supportive cares, as well as accurate follow up decisions.

\section{Objectives}

Due to the high prevalence and incidence of gastric cancer in Iran, especially in the northwest, this study was designed to find out the association between CDX2 expression and survival and other clinicopathological characteristics in patients with gastric cancer.

\section{Materials and Methods \\ Study design}

This was a longitudinal study to investigate the possible association between the expression of CDX2 and overall survival and clinicopathological characteristics in patients with gastric cancer. They were referred to the ward of hematology and oncology at Shahid Ghazi hospital (a hospital of Tabriz University of Medical Sciences, Tabriz, Iran). All the patients were studied from the time of gastric cancer diagnosis until September 2017. The median of follow up period was 25.6 months.

Patients with confirmed pathology of gastric adenocarcinoma were included in the study based on a convenient sampling method and the patients with incomplete medical records and those who were unavailable due to the lack of contact information and/or were reluctant to continue their treatment at this center, excluded from the study.

The expression of CDX2 was examined by the immunohistochemistry method in the formalin-fixed paraffin-embedded surgical specimens of gastric cancer, using FLEX, Monoclonal Mouse, Anti-Human CDX2, Clone DAK-CDX2, Ready-to-Use (Dako Autostainer/ Autostainer Plus) kit; Lot number: 10109695; REF: IS080, DAKO, Denmark in paraffin blocks, and demographic information as well as clinicopathological parameters were obtained from the patients' medical records. The required information included age, gender, body mass index, stage of the disease, metastasis status, pathology type of the tumor, patients' socio-economy status, serum levels of tumor markers carcinoembryonic antigen (CEA), cancer antigen 125 (CA-125), cancer antigen 19-9 (CA 19-9) and the status of death/survival. The final survival status of the patients was confirmed asking them by a telephone call.

\section{Ethical approval}

The study protocol was approved by the Ethics Committee of Tabriz University of Medical Sciences, Tabriz, Iran. (Approval ID: IR.TBZMED. REC.5/D/86142). The current study was performed according to the Institutional Committee for the Protection of Human Subjects, which was adopted by the 18th World Medical Assembly, Helsinki, Finland and its later amendments (Declaration of Helsinki). Written informed consents were obtained from all patients. This article is derived from Dr. Farideh Akbari's thesis on Hematology and Oncology fellowship course in Tabriz University of Medical Sciences.

\section{Statistical analysis}

Mean (Standard deviation) and frequency (\%) were used for summarizing numerical and categorical variables respectively. Survival probability was calculated and compared by Kaplan-Meier method and the log-rank test respectively. Cox regression analysis was conducted for evaluating the association between CDX2 expression and time to death, adjusting for confounding variables. In this analysis adjusted hazard ratio (HR) and 95\% Confidence Interval (CI) were presented. Fisher's exact test was used for assessing the relationship between clinicopathological variables and CDX2 expression. The significance level was set at 0.05 considering a two-tailed test. All statistical analyses were performed by SPSS software, version 17 (SPSS Inc., IL, Chicago, USA).

\section{Results}

In this study, 100 patients with gastric cancer were enrolled and 92 patients completed the study. The flow diagram of the study was shown in Figure 1. Thirty-six patients were female $(39 \%)$ and the rest were male $(61 \%)$. The mean age of the patients was 60.58 (11.88) year and the mean body mass index (BMI) was $23.13(4.95) \mathrm{kg} / \mathrm{m}^{2}$. General characteristics of the patients were summarized in Table 1 and clinicopathological parameters including stage of the disease, metastasis status, pathological type of gastric carcinoma, and history of patients' symptoms were shown in Table 2 .

There was not any statistically significant difference between two groups of patients (CDX2 positive versus negative) in terms of overall survival. One-year overall survival was $84 \%$ in CDX2 positive patients while it was $70 \%$ in the other group and three-year overall survival 


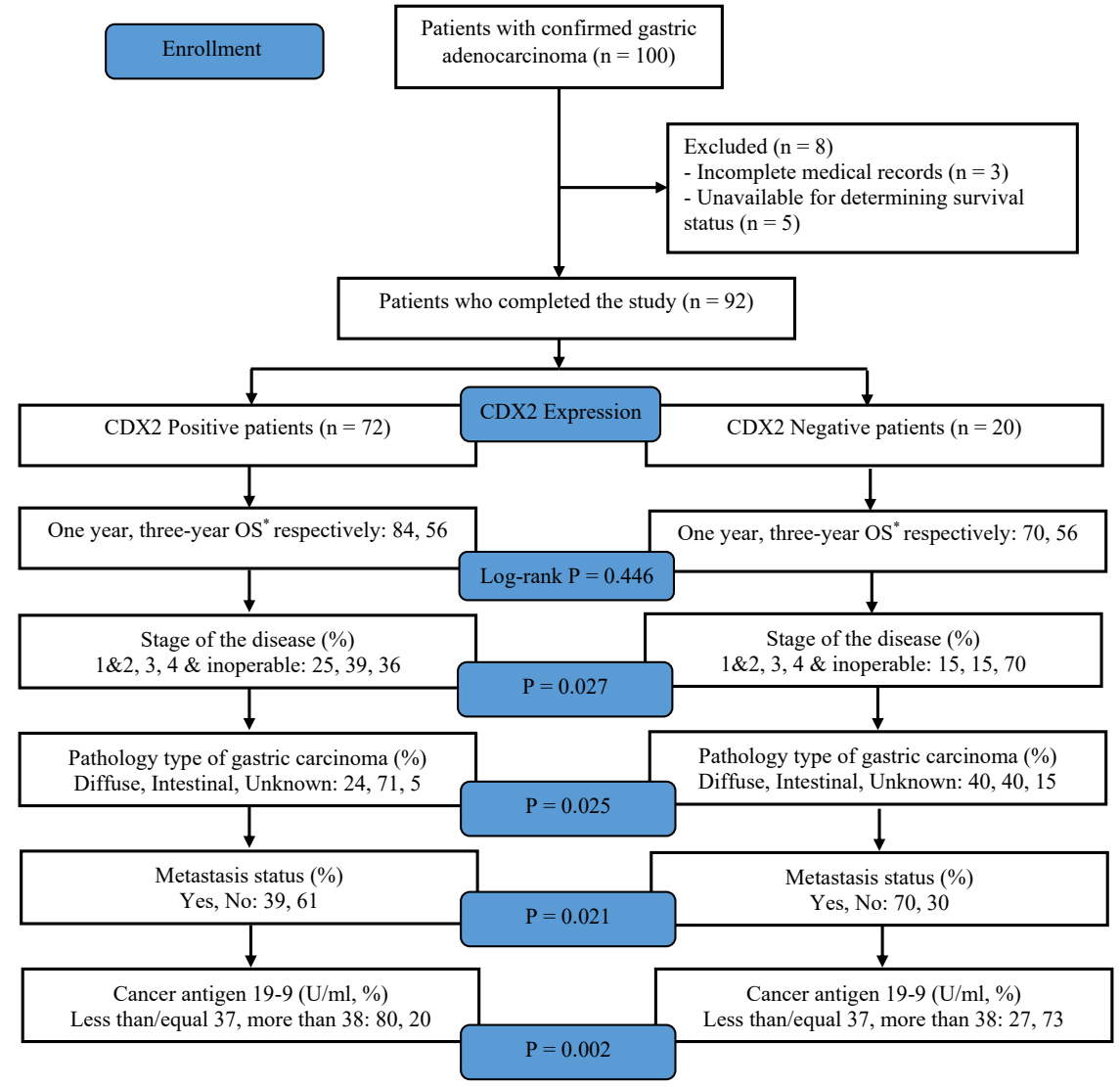

Figure 1. Flow Diagram of the study results. OS, overall survival.

was $56 \%$ in both groups of patients $(P=0.446)$ (Figure 2). However, the risk of death was $21 \%$ lower in CDX2 positive patients, after adjusting for age and gender $(\mathrm{HR}=$ $0.79,95 \%$ CI ( 0.35 to $1.77 ; P=0.565)$.

\section{The status of clinicopathological variables associated with CDX2 expression}

There was a significant difference between CDX2 positive and CDX2 negative patients regarding the stage of the disease, while $70 \%$ of CDX2 negative patients were at higher stages of disease as it was $36 \%$ in CDX2 positive patients $(P=0.027$; Table 3$)$. Moreover, in $70 \%$ of CDX2

Table 1. General characteristics of the study subjects

\begin{tabular}{lc}
\hline Variables & \\
\hline Age $\left(y\right.$, mean $\left.\pm \mathrm{SD}^{\mathrm{a}}\right)$ & $60.58 \pm 11.88$ \\
Gender, No. $(\%)$ & $36(39)$ \\
Male & $56(61)$ \\
Female & $23.13 \pm 4.95$ \\
$\mathrm{BMI}^{\mathrm{b}}\left(\mathrm{kg} / \mathrm{m}^{2}\right.$, Mean $\left.\pm \mathrm{SD}^{\mathrm{a}}\right)$ & $6(7)$ \\
Socioeconomic status, No. $(\%)$ & $59(64)$ \\
High & $27(29)$ \\
\hline Middle & \\
Low &
\end{tabular}

negative gastric cancer patients, metastasis was occurred, while, it was $39 \%$ in the other group. There was a significant difference in terms of pathology type of gastric carcinoma between these two groups. Seventy-one percent of CDX2 positive patients had intestinal type pathology while it was $40 \%$ in CDX2 negative subjects, all of these differences were statistically significant (Table 3 ).

Considerably, there was no statistically significant

Table 2. Clinicopathologic parameters of the patients

\begin{tabular}{ll}
\hline Variables & No. $(\%)$ \\
\hline Stage of disease & $21(23)$ \\
\hline $1 \& 2$ & $31(34)$ \\
3 & $40(43)$ \\
$4 \&$ Inoperable & \\
\hline Pathology type & $26(28)$ \\
\hline Diffuse & $59(64)$ \\
Intestinal & $7(8)$ \\
Unknown & \\
Metastasis status & $42(46)$ \\
Metastasis & $50(54)$ \\
No metastasis & \\
Symptoms & $19(21)$ \\
Gastrointestinal bleeding & $25(27)$ \\
Dysphasia & $48(52)$ \\
Other symptoms &
\end{tabular}


Table 3. Differences between CDX2 positive and CDX2 negative patients in terms of clinicopathological parameters

\begin{tabular}{lccl}
\hline \multirow{2}{*}{ Variables } & \multicolumn{2}{c}{ CDX2, No. (\%) } & \multirow{P}{P}{} \\
\cline { 2 - 3 } value $^{\mathrm{a}}$ & Positive $(\mathbf{n = 7 2})$ & Negative $(\mathbf{n = 2 0})$ & 0.027 \\
\hline Stage of disease & $18(25)$ & $3(15)$ & \\
1 \&2 & $28(39)$ & $3(15)$ & \\
3 & $26(36)$ & $14(70)$ & \\
4 \& Inoperable & & & \\
Pathology type & $17(24)$ & $9(45)$ & \\
Diffuse & $51(71)$ & $8(40)$ & \\
Intestinal & $4(5)$ & $3(15)$ & \\
Unknown & & & \\
\hline Metastasis status & $28(39)$ & $14(70)$ & \\
Metastasis & $44(61)$ & $6(30)$ & \\
No metastasis & & & \\
\hline
\end{tabular}

$P$ value was calculated based on Fisher's exact test.

difference between the two groups of CDX2 positive and CDX2 negative patients regarding following variables; age, gender distribution, BMI, CA125 and CEA $(P=0.570$, $0.197,0.653,0.380$ and 0.266 , respectively). However, patients of the two groups were statistically different in terms of CA.19-9 levels $(P=0.028)$.

\section{Discussion}

In this study, 92 patients with gastric cancer were assessed for expression of CDX2 in Cancerous tissue and the overall survival rate was compared between CDX2 positive and negative study subjects. Moreover, clinicopathological characteristics including stage of the disease, pathology type, metastasis status, and accompanying symptoms were examined in both CDX2 positive and negative patients. Based on the obtained results, CDX2 positive patients had a relatively higher one-year overall survival and a significant lower disease stage, Metastasis rate and associated symptoms.

Considering globally importance of gastric cancer as one of the main cancer-causing death with no strong prognostic factors, finding precise predictors is an urgent need in this group of the patients. CDX2 is a transcription factor known as a caudal-related homeobox protein, expressed in the intestinal cells and it has a crucial role in their proliferation and differentiation. Therefore, it is considered as a biological marker of intestinal metaplasia in initiation and progression of gastric cancer. However, the importance of CDX2 expression in the prognosis of gastric cancer was studied before with controversial results $(5,11,12,16,17)$.

In a study performed by Seno et al, survival was statistically longer in the CDX2 positive gastric tumor individuals with intestinal metaplasia than the CDX2 negative ones and $64 \%$ of the patients were CDX2 positive (11). Another study conducted by Bai et al, showed the same association between CDX2 expression and survival rate (16). In a study done by Qin et al, a significant negative association between the expression of CDX2 and

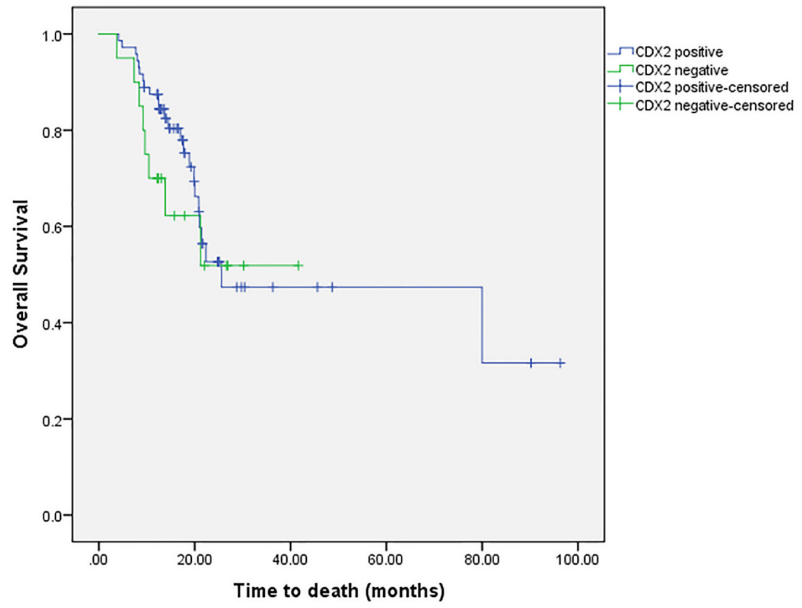

Figure 2. Comparison of survival rate between patients according to CDX2 expression. $\mathrm{P}$ value was calculated based on log rank test. The risk of death was $21 \%$ lower in CDX2 positive patients, after adjusting for age and gender $(\mathrm{HR}=0.79,95 \% \mathrm{Cl}: 0.35-1.77, P=0.565)$

metastasis status and stage of the disease was detected. Additionally, they found CDX2 positive patients had a longer survival than those who were CDX2 negative, came to a conclusion of being CDX2 positive was related to a better prognosis and higher rate of survival in patients with gastric cancer (10). This finding is in accordance with the results of the current study.

The prognostic importance of CDX2 expression for predicting outcomes in patients with gastric cancer was confirmed in other similar studies (18-20). Our results are in line with the latter, while both studies showed a positive association between CDX2 expression and a higher proportion of intestinal type carcinoma, less invasion and metastasis and also lower stage of the disease. In fact, these parameters are all related to a higher rate of survival in patients with gastric cancer. Some previous studies revealed a remarkably higher intestinal type gastric cancer than the diffuse type carcinoma in CDX2 positive patients with gastric cancer (15). Park et al concluded a tumor suppressor effect for CDX2 in these patients, as its expression decreases with the advancing stage of the disease (15). Interestingly, in the majority of the CDX2 positive patients of the current study, the levels of CA 19-9 was lower than $37 \mathrm{U} / \mathrm{mL}$. Although tumor markers are not strong prognostic factors, this finding is in favor of positive association between CDX2 expression and better outcomes.

Recently, a relationship between CDX2 expression and inflammation was detected. Chronic inflammation is highly associated with gastric carcinoma development and CDX2 expression is suppressed by activation of IL-6/ STAT 3 inflammation signaling pathway. On the other hand, CDX2 negative gastric carcinoma is associated with inactivation of P53 signaling pathway without any mutation in TP53, hence, in CDX2 negative patients with gastric cancer, P53 staining is negative. Taken together, 
CDX2 may be suggested as a useful prognostic factor for gastric cancer (21).

\section{Conclusion}

This study showed that Iranian gastric cancer patients with CDX2 positive expression had a less invasive pattern of gastric carcinoma and better outcomes. Obviously, finding strong prognostic factors in patients with gastric cancer is an immediate need. Likewise, it helps the oncologists for tailoring their medical decisions to the individual patients based on their characteristics.

\section{Limitations of the study}

One of the limitations of this study was that it was run in a single medical center caused to a relatively small sample size that maybe underlies nonsignificant statistical difference in terms of the survival between CDX2-positive and negative patients with gastric cancer. Additionally, assessing the co-expression of CDX2 and some biomarkers with prognostic value in gastric carcinoma like pancreatic duodenal homeobox-1 (PDX-1) (22), Phosphatase and Tensin Homolog (PTEN) (16), and osteopontin (17) may have more prognostic value, which we could not assess them due to funding limitations.

\section{Acknowledgements}

The authors thank all the patients who participated in the study and are also grateful to the Hematology and Oncology Research Center for helping with the study. We are also thankful to Dr. Ashraf Fakhrjou for her great help in laboratory measurements.

\section{Authors' contribution}

FA provided contributions to the design of the study and drafting the manuscript. ZG, AE contributed to the design of the study, analysis of data and drafting the manuscript. MAJ contributed to the analysis and the interpretation of the results. KR provided contributions to edition of the first draft, critical revision, and submitting the manuscript. BN performed the experiments and drafted the manuscript. All authors approved final draft of submitted version and took responsibility of all aspects of the work.

Conflicts of interest

The authors declared that there is no conflict of interest.

\section{Ethical considerations}

Ethical issues (including plagiarism, double publication) have been completely observed by the authors. This article does not contain any studies with animals performed by any of the authors.

\section{Funding/Support}

This article is resulted from Dr. Farideh Akbari's dissertation on Hematology and Oncology fellowship course in Tabriz University of Medical Sciences (Approval number: 95/5-6/4).

\section{References}

1. Sun P, Xiang JB, Chen ZY. Meta-analysis of adjuvant chemotherapy after radical surgery for advanced gastric cancer. Br J Surg. 2009;96:26-33. doi: 10.1002/bjs.6408.

2. Somi MH, Mirinezhad K, Farhang S, Jazayeri E, Sani A, SeifFarshad $M$, et al. Gastrointestinal cancer occurrence in East
Azarbaijan: a five year study from North Western Iran. Asian Pac J Cancer Prev. 2006;7:309-12.

3. Somi MH, Farhang S, Mirinezhad SK, Naghashi S, Seif-Farshad M, Golzari M. Cancer in East Azerbaijan, Iran: results of a population-based cancer registry. Asian Pac J Cancer Prev. 2008;9:327-30.

4. Somi MH, Golzari M, Farhang S, Naghashi S, Abdollahi L. Gastrointestinal cancer incidence in East Azerbaijan, Iran: update on 5 year incidence and trends. Asian Pac J Cancer Prev. 2014;15:3945-9.

5. Wang X-T, Wei W-Y, Kong F-B, Lian C, Luo W, Xiao Q, et al. Prognostic significance of $C \mathrm{~d} \times 2$ immunohistochemical expression in gastric cancer: a meta-analysis of published literatures. J Exp Clin Cancer Res. 2012;31:98. doi: 10.1186/1756-9966-31-98.

6. Mallo GV, Rechreche H, Frigerio JM, Rocha D, Zweibaum A, Lacasa $\mathrm{M}$, et al. Molecular cloning, sequencing and expression of the mRNA encoding human $\mathrm{Cdx} 1$ and $\mathrm{Cdx} 2$ homeobox. Down-regulation of $\mathrm{Cdx} 1$ and $\mathrm{Cd} \times 2$ mRNA expression during colorectal carcinogenesis. Int J Cancer. 1997;74:35-44.

7. Masood MA, Loya A, Yusuf MA. CDX2 as a prognostic marker in gastric cancer. Acta gastro-enterologica Belgica. 2016;79:197200.

8. Ge J, Chen Z, Wu S, Yuan W, Hu B, Chen Z. A clinicopathological study on the expression of cadherin-17 and caudal-related homeobox transcription factor (CDX2) in human gastric carcinoma. Clin Oncol. 2008;20:275-83. doi: 10.1016/j. clon.2008.01.013.

9. RuY, Zhang L, Chen Q, Gao SG, Wang GP, Qu ZF, et al. Detection and clinical significance of lymph node micrometastasis in gastric cardia adenocarcinoma. J Int Med Res. 2012;40:293-9. doi:10.1177/147323001204000129.

10. Qin R, Wang N-N, Chu J, Wang X. Expression and significance of homeodomain protein $\mathrm{Cdx} 2$ in gastric carcinoma and precancerous lesions. World J Gastroenterol. 2012;18:3296. doi: 10.3748/wjg.v18.i25.3296.

11. Seno H, Oshima M, Taniguchi M-A, Usami K, Ishikawa TO, Chiba T, et al. CDX2 expression in the stomach with intestinal metaplasia and intestinal-type cancer: Prognostic implications. Int J Oncol. 2002;21:769-74.

12. Xie Y, Li L, Wang X, Qin Y, Qian Q, Yuan X, et al. Overexpression of $\mathrm{Cd} 22$ inhibits progression of gastric cancer in vitro. Int J Oncol. 2010;36:509-16.

13. Almeida R, Silva E, Santos-Silva F, Siberg DG, Wang J, De Bolós $\mathrm{C}$, et al. Expression of intestine-specific transcription factors, CDX1 and CDX2, in intestinal metaplasia and gastric carcinomas. J Pathol.. 2003;199:36-40. doi: 10.1002/path.1246.

14. Joo MK, Park J-J, Chun HJ. Impact of homeobox genes in gastrointestinal cancer. World J Gastroenterol. 2016;22:8247. doi: 10.3748/wjg.v22.i37.8247.

15. Park DY, Srivastava A, Kim GH, Mino-Kenudson M, Deshpande V, Zukerberg LR, et al. CDX2 expression in the intestinal-type gastric epithelial neoplasia: frequency and significance. Mod Pathol. 2010;23:54. doi: 10.1038/modpathol.2009.135.

16. Bai Z, Ye Y, Chen D, Shen D, Xu F, Cui Z, et al. Homeoprotein $\mathrm{Cd} \times 2$ and nuclear PTEN expression profiles are related to gastric cancer prognosis. Apmis. 2007;115:1383-90. doi: 10.1111/j.1600-0463.2007.00654.x.

17. Zhang X, Tsukamoto T, Mizoshita T, Ban H, Suzuki H, Toyoda $\mathrm{T}$, et al. Expression of osteopontin and CDX2: indications of phenotypes and prognosis in advanced gastric cancer. Oncol Rep. 2009;21:609-13.

18. Mizoshita T, Tsukamoto T, Nakanishi H, Inada K, Ogasawara N, Joh T, et al. Expression of $\mathrm{Cdx} 2$ and the phenotype of advanced gastric cancers: relationship with prognosis. J Cancer Res Clin 
Oncol. 2003;129:727-34. doi: 10.1007/s00432-003-0499-6.

19. Fan Z, Li J, Dong B, Huang X. Expression of Cdx2 and hepatocyte antigen in gastric carcinoma: correlation with histologic type and implications for prognosis. Clin Cancer Res. 2005;11:616270. doi: 10.1158/1078-0432.CCR-05-0278.

20. Ha Kim G, Am Song G, Youn Park D, Han Lee S, Oh Kim $\mathrm{T}$, Jae Jo $\mathrm{H}$, et al. CDX2 expression is increased in gastric cancers with less invasiveness and intestinal mucin phenotype. Scand J Gastroenterol. 2006;41:880-6. doi: 10.1080/00365520500497140.
21. Saito M, Okayama H, Saito K, Ando J, Kumamoto K, Nakamura l, et al. CDX2 is involved in microRNA-associated inflammatory carcinogenesis in gastric cancer. Oncol Lett. 2017;14:6184-90. doi: 10.3892/ol.2017.6956.

22. Oz Puyan F, Can N, Ozyilmaz F, Usta U, Sut N, Tastekin E, et al. The relationship among PDX1, CDX2, and mucin profiles in gastric carcinomas; correlations with clinicopathologic parameters. J Cancer Res Clin Oncol. 2011;137:1749. doi: 10.1007/s00432-011-1044-7. 